\title{
How bad metals turn good: spectroscopic signatures of resilient quasiparticles
}

\author{
Xiaoyu Deng, ${ }^{1,2,3}$ Jernej Mravlje, ${ }^{4,1,5}$ Rok Žitko, ${ }^{5}$ Michel Ferrero, ${ }^{1}$ Gabriel Kotliar, ${ }^{3}$ and Antoine Georges ${ }^{4,1,6,2}$ \\ ${ }^{1}$ Centre de Physique Théorique, Ecole Polytechnique, CNRS, 91128 Palaiseau Cedex, France \\ ${ }^{2}$ Japan Science and Technology Agency, CREST, Kawaguchi 332-0012, Japan \\ ${ }^{3}$ Department of Physics, Rutgers University, Piscataway, NJ 08854, USA \\ ${ }^{4}$ Collège de France, 11 place Marcelin Berthelot, 75005 Paris, France \\ ${ }^{5}$ Jožef Stefan Institute, Jamova 39, Ljubljana, Slovenia \\ ${ }^{6} \mathrm{DPMC}$, Université de Genève, 24 quai Ernest Ansermet, CH-1211 Genève, Suisse
}

(Dated: October 30, 2018)

\begin{abstract}
We investigate transport in strongly-correlated metals. Within dynamical mean-field theory, we calculate the resistivity, thermopower, optical conductivity and thermodynamic properties of a hole-doped Mott insulator. Two well-separated temperature scales are identified: $T_{\mathrm{FL}}$ below which Landau Fermi liquid behavior applies, and $T_{\mathrm{MIR}}$ above which the resistivity exceeds the Mott-Ioffe-Regel value and 'bad-metal' behavior is found. We show that quasiparticle excitations remain well-defined above $T_{\mathrm{FL}}$ and dominate transport throughout the intermediate regime $T_{\mathrm{FL}} \lesssim$ $T \lesssim T_{\text {MIR }}$. The lifetime of these 'resilient quasiparticles' is longer for electron-like excitations, and this pronounced particle-hole asymmetry has important consequences for the thermopower. The crossover into the bad-metal regime corresponds to the disappearance of these excitations, and has clear signatures in optical spectroscopy.
\end{abstract}

The transport properties of metals with strong electron correlations are unconventional and poorly understood theoretically. Two facts regarding the temperature dependence of the resistivity are frequently observed. (i) Fermi-liquid (FL) behavior $\rho \propto T^{2}$ only holds below a temperature $T_{\mathrm{FL}}$ which is low compared to bare electronic energy scales. (ii) At high temperatures the resistivity is large and reaches values exceeding the MottIoffe-Regel (MIR) value. This 'bad-metallic' behavior 1 signals the breakdown of a quasiparticle (QP) description of transport, since the associated mean-free path $l$ would be smaller than the lattice spacing. This is observed in many materials: $\mathrm{Sr}_{2} \mathrm{RuO}_{4}$ has $T_{\mathrm{FL}} \simeq 20 \mathrm{~K}$, while the MIR value is reached at $T_{\mathrm{MIR}} \simeq 800 \mathrm{~K}$ (using $k_{F} l \sim 1$ as the MIR criterion); in $\mathrm{LiV}_{2} \mathrm{O}_{4}, T_{\mathrm{FL}}$ is a few degrees Kelvin, while $T_{\text {MIR }}$ is several hundreds, etc. (see [2, 3] for reviews). In two-dimensional organic materials, $T_{\mathrm{FL}}$ and $T_{\mathrm{MIR}}$ are closer but still distinct scales [4 6].

These observations raise the following questions. Why is $T_{\mathrm{FL}}$ much lower than $T_{\mathrm{MIR}}$ and what determines its value? Up to which temperature do QPs exist and what are the signatures of their disappearance? And, most importantly: how should one think of transport not only in the bad-metal, but also in the intermediate regime $T_{\mathrm{FL}} \lesssim T \lesssim T_{\mathrm{MIR}}$ where the resistivity does not follow Landau's $T^{2}$ behavior, but is still smaller than the MIR value? These questions also apply to cuprate superconductors, where the observation of quantum oscillations [7, 8] and $T^{2}$ behavior in transport [9, 10] and optics [11] have rejuvenated the relevance of FL states with a low $T_{\mathrm{FL}}$ (possibly with angular dependence along the Fermi surface).

In this article, we answer these questions in a particularly simple setting: a hole-doped Mott insulator described with dynamical mean-field theory (DMFT). Our most striking finding is that well-defined $Q P$ excitations survive well above the range of validity of FL theory. For over a decade in temperature above $T_{\mathrm{FL}}$ the transport can be accurately described in terms of these 'resilient quasiparticles' (RQPs). The high-temperature MIR crossover into the bad-metal regime is associated with their gradual extinction, which has a clear signature both in the singleparticle spectral function and in optical spectroscopy. In a hole-doped Mott insulator, the RQPs come with a strong particle-hole asymmetry: electron-like excitations are longer-lived than hole-like ones. This has direct consequences for the thermopower.

Previous DMFT work has investigated transport [12 16] and optical conductivity [6, 17, but not the precise temperature-dependence of the self-energy and of the momentum-resolved spectral function which reveals this intermediate RQP regime. We note that in the halffilled case relevant to organic compounds 4, 5, the hightemperature state is insulating-like, and hence the temperature window where this regime can be seen is narrower.

We solved the DMFT equations [18] for the holedoped Hubbard model using highly accurate numericalrenormalization group (NRG) [19, 20] and continuoustime quantum Monte Carlo [21, 22] techniques. In DMFT the real part of the optical conductivity reads

$$
\sigma(\omega)=\frac{2 \pi e^{2}}{\hbar} \int d \omega^{\prime} F_{\omega, \omega^{\prime}} \int d \epsilon \Phi(\epsilon) A_{\mathbf{k}}\left(\omega^{\prime}\right) A_{\mathbf{k}}\left(\omega^{\prime}+\omega\right)
$$

where $F_{\omega, \omega^{\prime}}=\left[f\left(\omega^{\prime}\right)-f\left(\omega+\omega^{\prime}\right)\right] / \omega$ with $f(\omega)$ the Fermi function. $A_{\mathbf{k}}(\omega)=-(1 / \pi) \operatorname{Im}\left[\omega+\mu-\varepsilon_{\mathbf{k}}-\Sigma(\omega)\right]^{-1}$ is the single-particle spectral function, with $\varepsilon_{\mathbf{k}}$ the energy of the state in the band, $\Sigma(\omega)$ the retarded self energy and $\mu$ the chemical potential. $\Phi(\varepsilon)=(1 / V) \sum_{\mathbf{k}}\left(\partial \varepsilon_{\mathbf{k}} / \partial \mathbf{k}_{x}\right)^{2} \delta(\varepsilon-$ $\left.\varepsilon_{\mathbf{k}}\right)$ contains the information about velocities. We used 

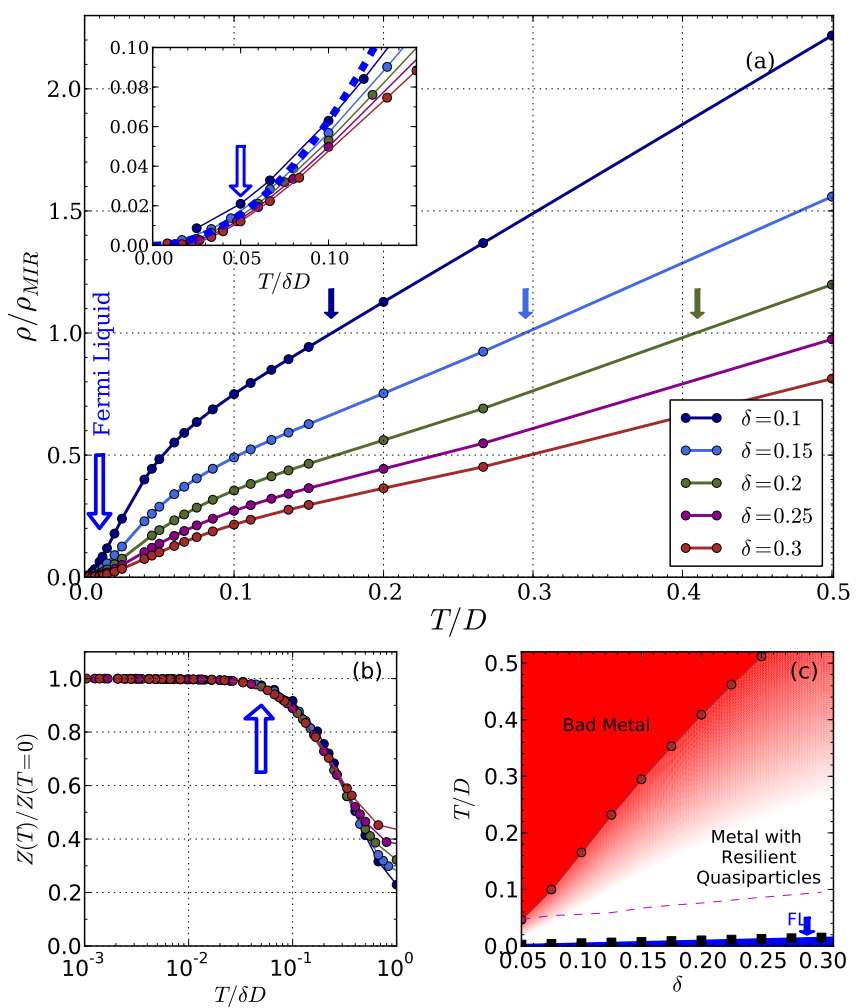

FIG. 1. (a) Temperature-dependence of the resistivity for several doping levels $\delta(U / D=4$, as in all figures). The MIR value as defined in the text is reached at a temperature $T_{\mathrm{MIR}}$ indicated by plain arrows. Inset: resistivity at low temperatures vs. $T / \delta D$ revealing the $T^{2}$ behavior (dashed line) below $T_{\mathrm{FL}}$ (empty arrow). (b) Determination of $T_{\mathrm{FL}}$ by a scaling plot of $Z(T) / Z(T \rightarrow 0)$ vs. $T / \delta D$. Here, $Z(T)^{-1} \equiv$ $1-\partial \operatorname{Re} \Sigma(\omega, T) / \partial \omega$. (c) The different regimes: FL (blue) for $T<T_{\mathrm{FL}}$, bad metal (red) and intermediate RQP regime. The crossover into the bad metal is gradual: the onset of red shading corresponds to the optical spectroscopy signatures discussed in the text, while the red points indicate where $\rho_{\mathrm{MIR}}$ is reached. The thin dashed line indicates the 'knee' in $\rho(T)$.

a semicircular density-of-states (DOS) with a half-width $D$, and the corresponding sum-rule preserving expression $\Phi(\epsilon)=\Phi(0)\left[1-(\epsilon / D)^{2}\right]^{3 / 2}$. In the following, resistivity will be expressed in units of the MIR value defined as $1 / \rho_{\mathrm{MIR}} \equiv e^{2} \Phi(0) / \hbar D$. This choice is consistent with the criterion $k_{F} l=1$ for a parabolic band in two dimensions, for which the conductivity $\sigma=\left(k_{F} l\right) e^{2} \Phi\left(\varepsilon_{F}\right) / \hbar \varepsilon_{F}=$ $\left(k_{F} l\right) e^{2} / h$.

Fig. 17 summarizes our main result: as a function of temperature, three distinct regions appear. At low $T<T_{\mathrm{FL}}$, FL behavior is found. At high temperature, the system is a bad metal with no quasiparticles (this is indicated by the shaded area on Fig. 1; , more details below). Between these two limits, there is an extended region with well-defined quasiparticles, but that do not obey FL behavior. The nature of this metal with 're-
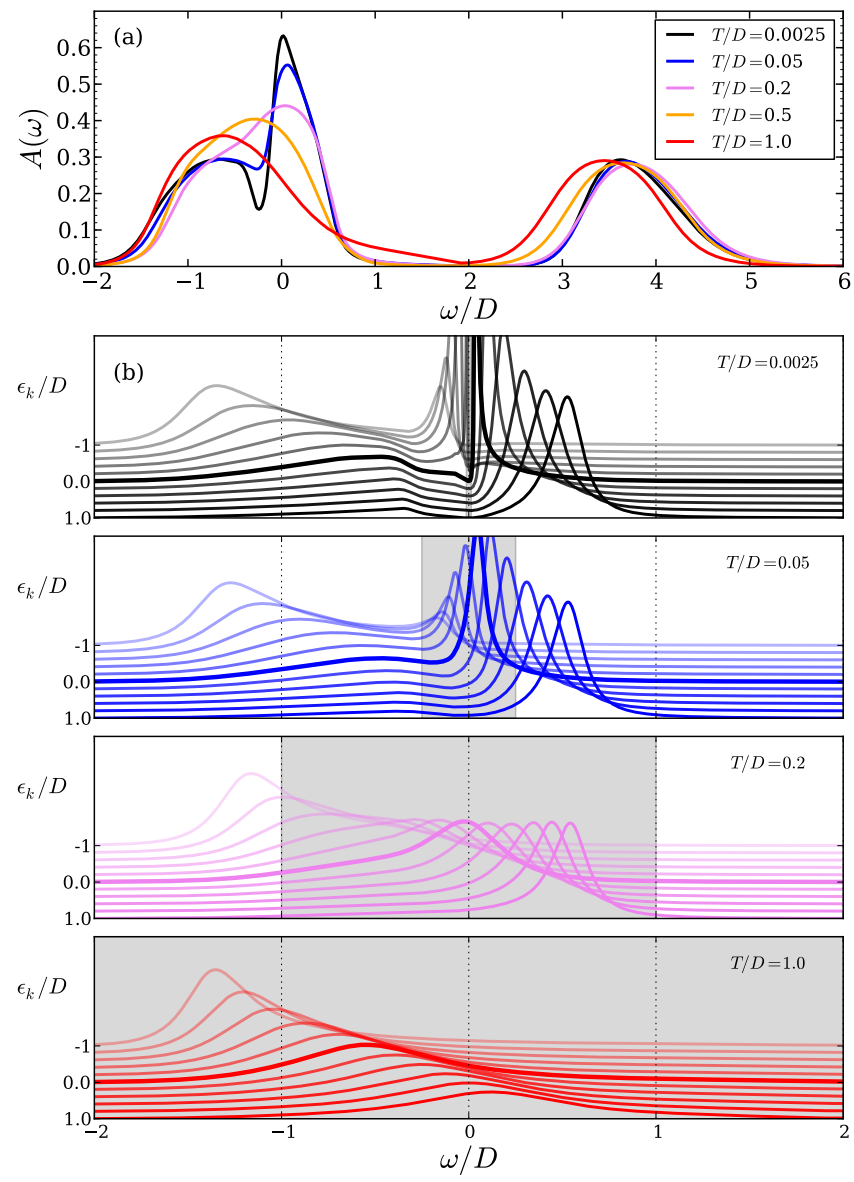

FIG. 2. (a) Temperature evolution of the total DOS for $\delta=0.2$. (b) Momentum- resolved spectral functions. The shaded area $\left[-5 k_{B} T, 5 k_{B} T\right]$ indicates the states with a significant contribution to transport.

silient quasiparticles' (RQPs) is the central focus of our work.

Let us first discuss transport (resistivity vs. T, Fig. 1a) in the light of these regimes. At low temperature the resistivity has a FL $T^{2}$ behavior. This extends up to a temperature $T_{\mathrm{FL}}$ (see inset in Fig. 1 1 ) which is proportional to the doping level, $T_{\mathrm{FL}} \simeq 0.05 \delta D$. Note that $T_{\mathrm{FL}}$ can be determined by other complementary criteria, like the scaling of $\operatorname{Im} \Sigma(\omega, T) / T^{2}$ vs. $\omega / T$ (see on-line supplementary [23]) or the scaling of $Z(T=0) / Z(T)$ vs. $T / \delta D$ (Fig. 1 $\mathrm{b}$ ). Above $T_{\mathrm{FL}}$, the resistivity increases approximately linearly (with a negative intercept). A knee-like feature is observed at a temperature $T_{*}$, above which the high- $T$ regime gradually sets in. $\rho(T)$ then has a linear temperature dependence (with a positive intercept), as can be shown from a high- $T$ expansion [15, 16, and smoothly crosses $\rho_{\mathrm{MIR}}$ (see arrows in Fig. 17) at a temperature $T_{\mathrm{MIR}} \sim 2 \delta D$.

The data therefore show that there is a wide temperature range in which transport does not follow the $T^{2}$ FL behavior, although the resistivity is still substantially 
smaller than $\rho_{\mathrm{MIR}}$. This raises the following question: what are the charge carriers in this intermediate metallic regime? To this aim, we depict in Fig. 2 the momentumresolved spectral function $A_{\mathbf{k}}(\omega)$ at selected temperatures as energy distribution curves $\left(A_{\mathbf{k}}(\omega)\right.$ vs. $\omega$ for different $\varepsilon_{\mathbf{k}}$ 's as well as the momentum-integrated DOS). These results reveal a remarkable fact: well-defined QP excitations exist throughout this intermediate regime, way above the FL scale. Our definition of the term 'quasiparticle' is a pragmatic one: we mean that $A_{\mathbf{k}}(\omega)$ displays a well-resolved peak in the vicinity of the Fermi level, in addition to a lower Hubbard band (LHB) and an upper Hubbard band (UHB).

For $T<T_{\mathrm{FL}}(T / D=0.0025$ in Fig. 2) sharp peaks are seen close to the Fermi energy $(\omega=0)$, characteristic of long-lived Landau QPs. For $T>T_{\mathrm{FL}}(T / D=0.05$ curves), the peaks broaden and the RQPs are visible mostly on the unoccupied side of the FS $(\omega>0)$. As the temperature increases (see e.g. $T / D=0.2$ ), the QP can barely be resolved (see [23], for a color map representation) and eventually disappear, with just the Hubbard satellites remaining in the spectra (e.g. the two-peak structure that is visible in the total DOS of Fig. 2a at $T / D=0.2$ is not present for $T / D=0.5$ anymore). This crossover into the bad-metallic regime is a gradual one and there is not a precise temperature where the QP suddenly die out (below we discuss how the optical conductivity provides a criterion for the onset of the badmetallic behavior). Our data nevertheless clearly show that they still exit well above $T_{*}$ and that they have completely disappeared at $T_{\mathrm{MIR}}$.

$T_{\mathrm{FL}}$ and $T_{\mathrm{MIR}}$ appear as overall scales between which RQPs exist. Both these temperatures are proportional to the doping level $\delta$ but with very different prefactors $T_{\mathrm{FL}} / T_{\mathrm{MIR}} \simeq 0.025$. Correspondingly, the resistivity at $T_{\mathrm{FL}}$ is much smaller than $\rho_{\mathrm{MIR}}, \rho\left(T_{\mathrm{FL}}\right) / \rho_{\mathrm{MIR}} \simeq 0.016$ (a low- $T$ expansion in the FL region yields $\rho(T) / \rho_{\mathrm{MIR}} \sim$ $\left.6.3(T / \delta D)^{2}+\cdots\right)$. Let us emphasize that the BrinkmanRice scale $\delta D$, which is a measure of the kinetic energy of QPs and hence of the quantum degeneracy scale, is associated with $T_{\mathrm{MIR}}$, not with $T_{\mathrm{FL}}$.

Examination of the self-energy (Fig. 3) helps understanding the nature of the QP excitations, as well as of the different transport regimes. In local DMFT, $-Z \operatorname{Im} \Sigma$ can be interpreted at low- $\omega$ as the inverse of the QP lifetime, and $-\operatorname{Im} \Sigma$ as the transport scattering rate. Fig. 3b displays $\operatorname{Im} \Sigma(\omega, T)$ vs. $T$ for different excitation energies $\omega$. At $\omega=0$ (thick curve), FL behavior $\operatorname{Im} \Sigma \sim T^{2}$ applies at low- $T$, corresponding to very long-lived QPs. Note that strict FL behavior breaks down already below the temperature at which $-Z \operatorname{Im} \Sigma(0, T) \sim T$ [24]. At finite frequency, the hole-like excitations have higher scattering rate than electron-like ones (Fig. 3a,b). At $T_{*} \simeq 0.08 D$, the curves of $\operatorname{Im} \Sigma v s . T$ for different positive $\omega$ 's display a crossing point. Above $T_{*}$, the scattering rate is a decreasing function of frequency: low-energy electron-like exci-

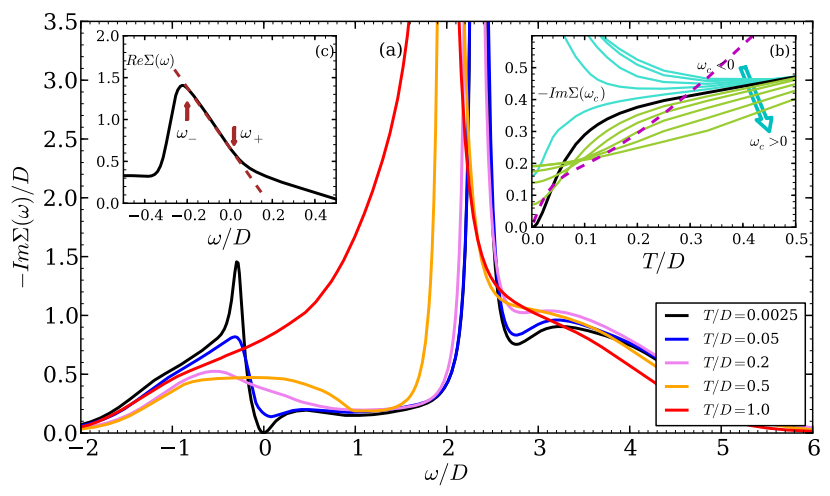

FIG. 3. Self-energy and particle-hole asymmetry. (a) $\operatorname{Im} \Sigma(\omega)$ for different temperatures. (b) Temperature dependence of $\operatorname{Im} \Sigma\left(\omega_{c}\right)$ for $\omega_{c}=-0.5,-0.4, \ldots,-0.1$ (turquoise), $\omega_{c}=0.0$ (thick black) and $\omega_{c}=0.1,0.2, \ldots, 0.5$ (green). Below the dashed line $Z(T) \operatorname{Im} \Sigma \lesssim T$. (c) $\operatorname{Re} \Sigma(\omega)$ at $T / D=0.0025$, and the two 'kinks' (arrows).

tations with finite $\omega>0$ have a longer lifetime than those at $\omega=0$. These finite-energy $\omega>0$ excitations provide the largest contribution to conductivity in the intermediate RQP regime [25]. Their inverse lifetime depends weakly on temperature for $T>T_{*}$ (almost saturated behavior in Fig. 3b ) and remains much smaller than the bandwidth and at most comparable to $k_{B} T$. For early considerations on a $\mathrm{QP}$ description of transport beyond the FL regime in the context of electron-phonon interactions, see [26].

The strong electron-hole asymmetry has also other interesting consequences. Because in the RQP regime the $\omega<0$ states are strongly damped, the Fermi surface as determined by the maximum intensity of $A_{\mathbf{k}}(\omega=0)$ 'inflates' to a larger volume than the $T=0$ Luttinger volume 23. From Fig. 3]c, it is also seen that the deviation from linearity of $\operatorname{Re} \Sigma\left(\sim \Sigma_{0}+\omega(1-1 / Z)\right.$ at low $\omega$ ) defines two distinct energy scales, $-\omega_{-}$for hole-like and $\omega_{+}$for electron-like excitations, leading to 'kinks' in QP dispersions, as documented by previous studies [27, 28]. We note that the smallest kink energy $\omega_{+} \ll \omega_{-}$sets the scale for deviations from FL behavior $\left(\omega_{+} \simeq \pi T_{\mathrm{FL}}\right)$. Using quite different theoretical methods, previous studies [29, 30] have also emphasized the importance of particle-hole asymmetry in hole-doped Mott insulators.

A sensitive probe of the particle-hole asymmetry is the Seebeck coefficient (thermopower) $Q(T)$ shown on Fig. 4 . Strikingly, the subleading particle-hole asymmetric terms in the low-frequency expansion of $\operatorname{Im} \Sigma$ modify the slope of $Q(T)$ at low- $T$ by a factor of about two, as compared to a naive FL theory estimate (thick dashed line) that would only retain terms $\sim \omega^{2}+(\pi T)^{2}$. This effect was anticipated in Ref. 31 and is shown here to be quantitatively important. The near saturation of the scattering rate of the RQPs, discussed above, is also responsible 


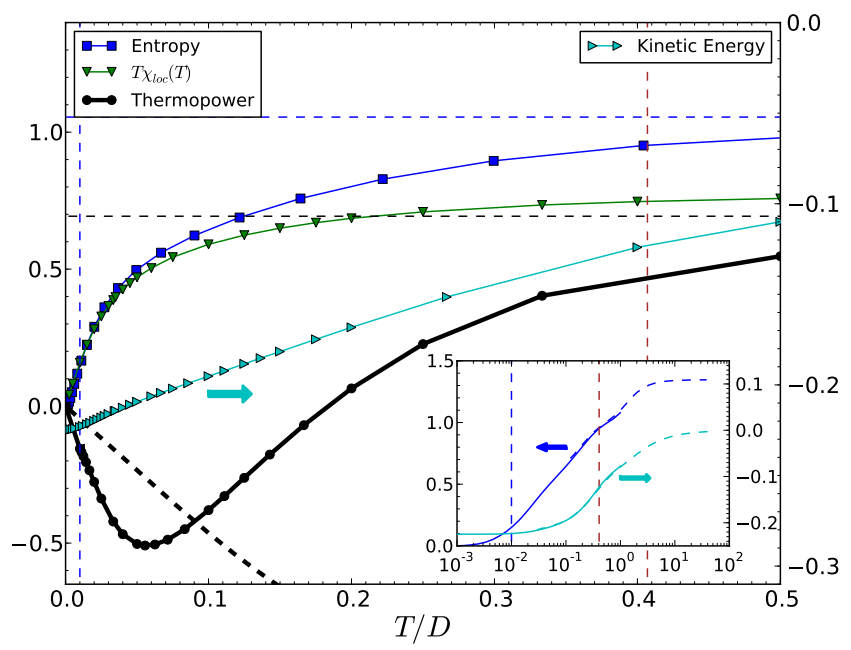

FIG. 4. Entropy (in units of $\left.k_{B}\right), T \chi_{\text {loc }}\left(\mu_{B}=1\right.$ ), and Seebeck coefficient (in units of $k_{B} /|e|$ ), for $\delta=20 \%$. Horizontal dashed lines indicate the atomic (Heikes) limit for the entropy and thermopower. Vertical dashed lines denote $T_{\mathrm{FL}}$ and $T_{\mathrm{MIR}}$. The thermopower at low- $T$ is compared to the FL estimate (thick dashed) in which the particle-hole asymmetry of $\operatorname{Im} \Sigma$ is neglected. Kinetic energy in units of $D$ (right scale). Inset: Entropy and kinetic energy versus temperature on a log scale.

for $Q(T)$ still increasing in an electron-like manner up to $T \simeq T_{*}$. At a higher temperature within the RQP regime $Q(T)$ changes sign and, when entering the badmetal regime, approaches the simple Heikes estimate for $D \lesssim T \ll U$ (Fig. 4]). The atomic Kelvin formula [23, 32 ] successfully describes the thermopower there, which can thus be taken as another fingerprint of a bad-metal. The accuracy of approximate formulas for thermopower has been tested also in other studies [33, 34.

It is interesting to observe how the different transport regimes relate to thermodynamic observables. On Fig. 4,we display the entropy $S(T)$, the kinetic energy $K(T)$, and the Curie constant $T \chi_{\text {loc }}$ associated with the local (q-integrated) magnetic susceptibility. The entropy as well as the Curie constant reach remarkably high values already below $T_{\mathrm{MIR}}$. In the RQP regime, the system thus has to be thought of in terms of two fluids: a mixture of local moments and of the resilient QP states. Above $T_{\mathrm{MIR}}$, the entropic contribution to the free energy overcomes the kinetic energy gain. The system is fully incoherent, its entropy approaches the atomic limit and the Curie constant saturates.

Optical spectroscopy (Fig. 5) can be used to detect the two crossovers, between the FL and the RQP regime and from the latter into the bad-metal. For $T<T_{\mathrm{FL}}, \sigma(\omega)$ displays a narrow low-frequency peak which decays as $1 / \omega^{2}$. This Drude peak corresponds to optical transitions involving only $\mathrm{QP}$ states and has a spectral weight proportional to doping level $\delta$. In the FL regime, the Drude peak is well separated from a higher frequency 'hump' (at

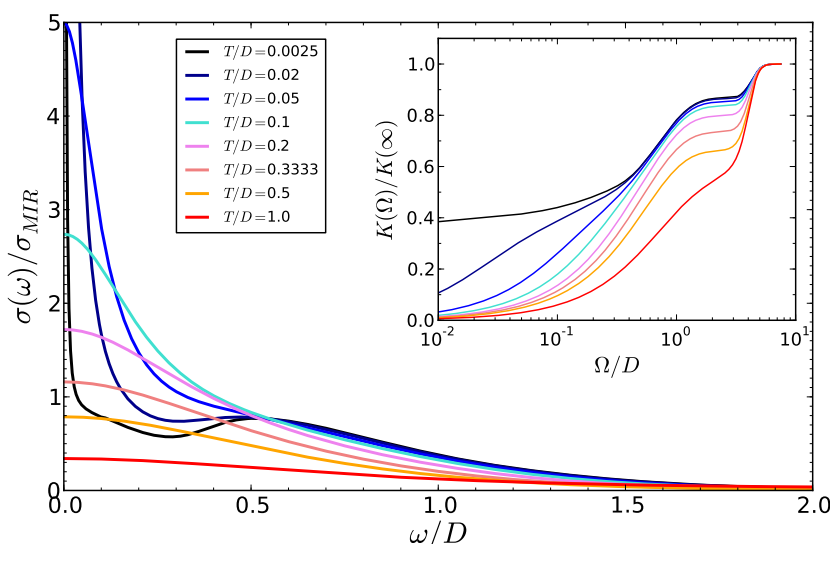

FIG. 5. Optical conductivity for $\delta=20 \%$. (Inset:) Optical spectral weight integrated up to $\Omega$, normalized to the kinetic energy.

$\omega / D \sim 0.5$ in Fig. 5) which corresponds to transitions between the LHB and QP states [17. The distance of the LHB to the Fermi level, of order $\mu$ (a fraction of the bandwidth) sets the energy scale for these transitions. This typically corresponds to the mid-infrared range in narrow-band correlated materials. The crossover out of the FL regime into the 'resilient metal' regime leads to a broader low-frequency peak whose frequency dependence is no longer $1 / \omega^{2}$. For $T / D=0.02 \sigma(\omega)$ can be fit to $1 / \omega^{\alpha}$ with $\alpha \approx 1.2$ for frequencies $0.02 \lesssim \omega / D \lesssim 0.2$. The low- $T$ data display an interesting 'non-Drude foot' with weak frequency dependence of $\sigma(\omega)$ between the Drude and the mid-infrared peaks. Above $T_{*}$ the Drude and mid-infrared features merge.

For a rather extended temperature range into the RQP regime, the spectral weight redistribution upon increasing $T$ takes place essentially entirely between the lowenergy QP states and the 'mid-infrared' feature, see inset of Fig. 5. For $T \gtrsim T_{*}$ (warmer part of the intermediate regime), some spectral weight transfer to higher frequencies starts taking place as well.

The beginning of the crossover into the bad-metal regime is signaled by two changes in $\sigma(\omega)$ happening at the same temperature of order $\delta D$ (it is shown as the onset of shading in Fig. 1k, which allows to have a clear view of the region where well-defined quasiparticles exist). First, the isosbestic crossing point (at $\omega / D \simeq 0.5$ ) is lost and the low-frequency non-Drude peak is replaced by a very broad peak, which corresponds to transitions involving only the LHB. Second, spectral weight is now redistributed over a considerable energy range, extending all the way to the UHB (inset). In the bad-metal, the kinetic energy (to which $\sigma(\omega)$ integrates) is strongly dependent on temperature (Fig. 4) and the broad peak has correspondingly a height which continues diminishing with $T$, which implies that the resistivity does not saturate [2]. 
In summary, our study reveals that resilient QP excitations persist well above $T_{\mathrm{FL}}$. They control transport properties until they disappear at a temperature roughly of order $T_{\mathrm{MIR}}$. The coexistence of QP states with localized magnetic moments which carry large entropy is intriguing, and demands closer theoretical investigation including antiferromagnetic correlations beyond DMFT. For hole-doped Mott insulators, a pronounced particlehole asymmetry is found. This calls for new momentumresolved spectroscopic probes which would be able to access the 'dark side' of the Fermi surface and for closer investigations of the electron-doped materials where the signatures of this regime could be seen using conventional ARPES.

We would like to thank C. Berthod, L. de' Medici, M. Dressel, A. Millis, T. V. Ramakrishnan, A. M. Tremblay, D. van der Marel, and especially N. Hussey and S. Shastry for very useful discussions, and Wenhu Xu for exchanges about thermodynamic observables. This work was partially supported by ICAM (X.D.) and the Swiss National Science Foundation MaNEP program. R.Ž. was supported by ARRS under program P1-0044.

Note added: During completion of this manuscript, related results regarding the thermopower were independently reported [35].

[1] V. J. Emery and S. A. Kivelson, Phys. Rev. Lett. 74, 3253 (1995)

[2] N. Hussey, K. Takenaka, and H. Takagi, Phil. Mag. 84, $2847(2004)$

[3] O. Gunnarsson, M. Calandra, and J. E. Han, Rev. Mod. Phys. 75, 1085 (2003)

[4] J. Merino and R. H. McKenzie, Phys. Rev. B 61, 7996 (2000)

[5] P. Limelette, P. Wzietek, S. Florens, A. Georges, T. A. Costi, C. Pasquier, D. Jérome, C. Mézière, and P. Batail, Phys. Rev. Lett. 91, 016401 (2003)

[6] J. Merino, M. Dumm, N. Drichko, M. Dressel, and R. H. McKenzie, Phys. Rev. Lett. 100, 086404 (2008)

[7] N. E. Hussey, M. Abdel-Jawad, A. Carrington, A. P. Mackenzie, and L. Balicas, Nature 425, 814 (2003)

[8] N. Doiron-Leyraud, C. Proust, D. LeBoeuf, J. Levallois, J.-B. Bonnemaison, R. Liang, D. A. Bonn, W. N. Hardy, and L. Taillefer, Nature 447, 565 (2007)

[9] N. E. Hussey, Journal of Physics: Condensed Matter 20, 123201

[10] M. Greven and N. Barišić(2012), internal communication

[11] S. I. Mirzaei, D. Stricker, J. N. Hancock, C. Berthod, A. Georges, E. van Heumen, M. K. Chan, X. Zhao, Y. Li, M. Greven, N. Barišić, and D. van der Marel, "Evidence for a fermi liquid in the pseudogap phase of high-tc cuprates," ArXiv:1207.6704

[12] T. Pruschke, D. Cox, and M. Jarrell, Europhys. Lett. 21, 593 (1993)

[13] M. Jarrell and T. Pruschke, Phys. Rev. B 49, 1458 (1994)

[14] H. Kajueter, G. Kotliar, and G. Moeller, Phys. Rev. B 53, 16214 (1996)
[15] G. Pálsson and G. Kotliar, Phys. Rev. Lett. 80, 4775 (1998)

[16] G. Palsson, Computational studies of thermoelectricity in strongly correlated electron systems, Ph.D. thesis, Rutgers University, NJ (2001)

[17] M. Jarrell, J. K. Freericks, and T. Pruschke, Phys. Rev. B 51, 11704 (1995)

[18] A. Georges, G. Kotliar, W. Krauth, and M. J. Rozenberg, Rev. Mod. Phys. 68, 13 (1996)

[19] R. Bulla, T. A. Costi, and T. Pruschke, Rev. Mod. Phys. 80, 395 (2008)

[20] R. Žitko, "NNRG ljubljana" - open source numerical renormalization group code," http://nrgljubljana. ijs.si/

[21] E. Gull, A. J. Millis, A. I. Lichtenstein, A. N. Rubtsov, M. Troyer, and P. Werner, Rev. Mod. Phys. 83, 349 (2011)

[22] M. Ferrero and O. Parcollet, "TRIQS: a Toolbox for Research on Interacting Quantum Systems," http://ipht. cea.fr/triqs

[23] "Online supplementary material,"

[24] J. Mravlje, M. Aichhorn, T. Miyake, K. Haule, G. Kotliar, and A. Georges, Phys. Rev. Lett 106, 096401 (2011)

[25] For $T \lesssim T_{\text {MIR }}$, the dc conductivity from (1) can be approximated by a generalized Drude-like formula $\sigma_{d c} \propto$ $\int d \omega f^{\prime}(\omega) \Phi\left(\epsilon_{\omega, T}^{*}\right) / \operatorname{Im} \Sigma(\omega)$ with $\epsilon_{\omega, T}^{*} \equiv \mu+\omega-\operatorname{Re} \Sigma$. This approximation is highly accurate up to $T_{*}$. The $\omega>0$ range provides the largest contribution in the RQP regime.

[26] R. E. Prange and L. P. Kadanoff, Phys. Rev. 134, A566 (1964)

[27] K. Byczuk, M. Kollar, K. Held, Y.-F. Yang, I. A. Nekrasov, T. Pruschke, and D. Vollhardt, Nat. Phys. 3, 168 (2007)

[28] P. Grete, S. Schmitt, C. Raas, F. B. Anders, and G. S. Uhrig, Phys. Rev. B 84, 205104 (2011)

[29] M. M. Zemljič, P. Prelovšek, and T. Tohyama, Phys. Rev. Lett. 100, 036402 (2008)

[30] B. S. Shastry, Phys. Rev. Lett. 109, 067004 (2012)

[31] K. Haule and G. Kotliar, in Properties and Applications of Thermoelectric Materials, NATO Science for Peace and Security Series B: Physics and Biophysics, edited by V. Zlatić and A. C. Hewson (Springer Netherlands, 2009) pp. 119-131

[32] M. R. Peterson and B. S. Shastry, Phys. Rev. B 82, 195105 (2010)

[33] M. Uchida, K. Oishi, M. Matsuo, W. Koshibae, Y. Onose, M. Mori, J. Fujioka, S. Miyasaka, S. Maekawa, and Y. Tokura, Phys. Rev. B 83, 165127 (2011)

[34] W. Xu, C. Weber, and G. Kotliar, Phys. Rev. B 84, 035114 (2011)

[35] L.-F. Arsenault, B. S. Shastry, P. Sémon, and A.M. S. Tremblay, "Entropy, frustration and large thermopower of doped mott insulators on the fcc lattice," ArXiv:1209.4349 


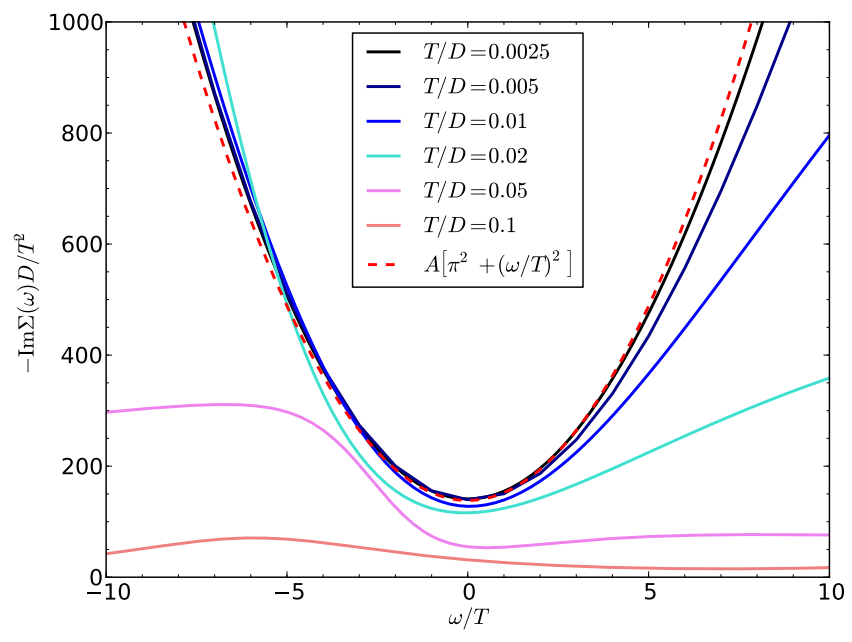

FIG. 6. The self energy scaling as $\omega / T$ for the doped Hubbard model considered in the main text (Bethe lattice, $U=4 D$, doping $\delta=0.2$ ). At low temperatures self energy follows Eq. A.2. $A$ is found to be close to $Z^{-2} \sim \delta^{-2}$. (For doping $0.2, Z=0.22$.)

\section{Supplementary information}

\section{Self-energy scaling}

In a Fermi liquid, $\operatorname{Im} \Sigma(\omega, T) \propto\left[\omega^{2}+(\pi T)^{2}\right]$ and therefore the self-energy obeys the following scaling in $\omega / T$ :

$$
-\frac{D \operatorname{Im} \Sigma}{T^{2}}=A\left[\pi^{2}+(\omega / T)^{2}\right] .
$$

In Fig. 6, we show $-\operatorname{Im} \Sigma(\omega, T) / T^{2}$ as a function of $\omega / T$ for different temperatures. As the temperature is lowered below $T_{\mathrm{FL}} \approx 0.01 D$, the curves collapse on a parabola, confirming the expected Fermi-liquid scaling law. Finding such a scaling is actually a stringent test on the numerical data. Very precise quantum Monte Carlo data (analytically continued with Padé approximants) and a strict control of the chemical potential were needed to obtain these results.

As the temperature is raised, the positive-frequency side quickly deviates from the scaling form, revealing a particle-hole asymmetry already for temperatures above $T / D=0.005$. The deviations appear at a scale $\omega_{+} \approx$ $\pi T_{\mathrm{FL}}$. On the negative-frequency side the self-energy follows the quadratic behavior much more robustly and deviates from the scaling function only at about $T / D=$ 0.02 . Notice that the corrections on the $\omega>0$ side grow linearly with temperature. This leads to a Seebeck coefficient which is linear in $T$ at low temperatures, but with an enhanced slope as compared to the result one would get if these corrections were neglected.

The transport probes an energy window of a few (say from -5 to 5$) k_{B} T$. In this energy window the self-energy starts to deviate appreciably at $T / D=0.01$. This is where the resistivity (within the precision of our data) visually departs from the $T^{2}$ law.

\section{Temperature evolution of momentum-resolved spectra}

In Fig. 7 we plot the temperature evolution of the momentum-resolved spectra using a color-map where bright (dark) colors indicate high (low) values of $A_{k}(\omega)$.

At low temperatures, the data display two peaks corresponding to: the lower Hubbard band (LHB) which disperses around $\omega_{\mathrm{LHB}} \sim-\mu_{0}$ and the quasiparticle peak (QP) in the vicinity of $\omega \sim 0 . \mu_{0}$ is the effective chemical potential at $T=0$. The upper Hubbard band (UHB) centered at the energy $\omega_{\mathrm{UHB}} \sim U-\mu_{0}$ lies above the energy range displayed in the plot.

The lowest temperature data $T / D=0.0025$ show a very sharp QP peak around $\omega=0$, which is rapidly broadened as the frequency is increased. At very small frequencies, the slope of the dispersion is found to be approximately 5 times smaller than the bare one, as dictated by $Z \approx 0.2$. On the negative-frequency side this holds almost until the bottom of the band. On the positive-frequency side, instead, the kink at $\omega_{+}$is rapidly encountered. Above this kink, the slope of the band dispersion increases to about half the bare dispersion slope. The LHB, seen clearly for occupied states $k<k_{F}$, disperses at a slope close to that of the bare dispersion.

As the temperature is increased, the QP band broadens and becomes more dispersing. It becomes therefore progressively more difficult to resolve it from the LHB band. Nevertheless, for temperatures well above $T_{\mathrm{FL}}$ and $T_{*}$ (four leftmost panels) one can still clearly distinguish the QP band from the LHB. The maximum of the spectra is also indicated (lines). This maximum has a discontinuity at a point where the maximal value in the QP band becomes larger than the maximal value reached in the LHB band. Dashed and solid lines are used to denote the maximum in the QP and LHB band, respectively. Above $T / D=0.2$ the maximal value does not have a discontinuity anymore and the signature of the quasiparticles is only visible as a kink in the dispersion. This marks the onset of the bad-metal regime. Note that $T / D=0.2=\delta$ corresponds to the Brinkman-Rice scale. At the highest temperature $T / D=1.0$ the kink is not seen anymore.

The QP band crosses the Fermi energy at different momenta as the temperature increases. Identifying the Fermi surface with the momenta at which the spectral intensity of the QP band is maximal leads to the conclusion that the Fermi volume inflates as the temperature is increased. Note that the number of particles is fixed so that with this identification of the Fermi surface the Luttinger theorem is only obeyed at very low temperatures.

To elaborate on this, we plot on Fig. 8 the momentum- 

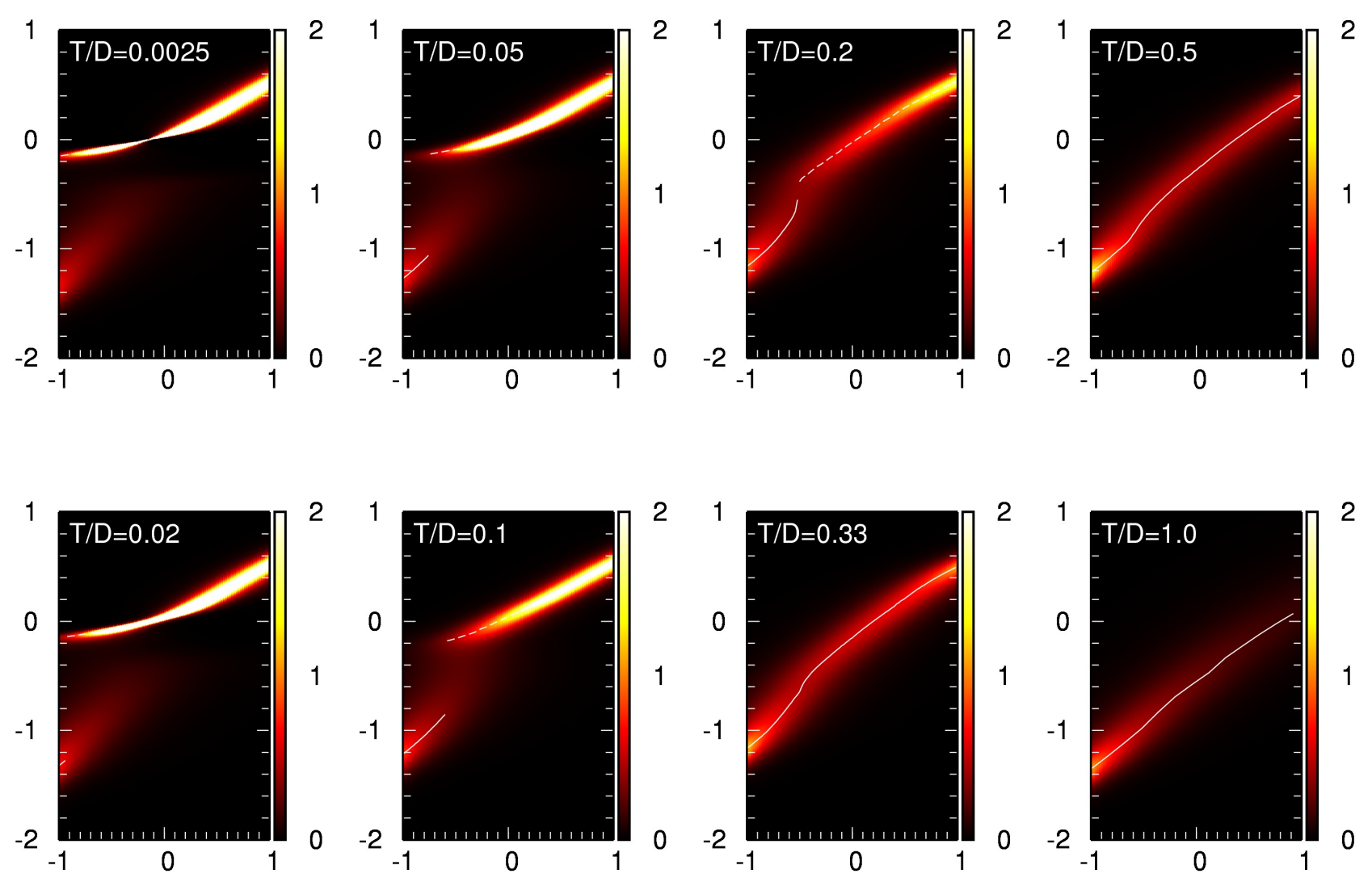

FIG. 7. Contour map of momentum-resolved spectra $A\left(\epsilon_{k}, \omega\right)$ at various temperatures for the same parameters as the data in the main text: doping $\delta=0.2$ and $U / D=4.0$.

distribution curve at $\omega=0$,

$$
A\left(\epsilon_{k}, 0\right)=\frac{1}{\pi} \frac{-\operatorname{Im} \Sigma(0, T)}{\left(\mu-\operatorname{Re} \Sigma(0, T)-\epsilon_{k}\right)^{2}+(\operatorname{Im} \Sigma(0, T))^{2}}
$$

for several temperatures. This spectral function has the shape of a Lorentzian centered at $\mu-\operatorname{Re} \Sigma(0, T)-\epsilon_{k}$ with a width $\operatorname{Im} \Sigma(0, T)$. At very low temperatures a sharp peak lies at the chemical potential, fulfilling the Luttinger theorem. When the temperature increases, the peak moves to higher momenta.

An alternative way to track this change is to look at the renormalized chemical potential $\mu_{\text {eff }}=\mu-\operatorname{Re} \Sigma(0, T)$ as a function of the temperature as shown in Fig. 9. In the Fermi-liquid regime, $\mu_{\text {eff }}$ essentially follows the noninteracting chemical potential $\mu_{0}$ (shown with a dashed line). At higher temperatures $\mu_{\text {eff }}$ rapidly increases.

An important lesson here is that observing a welldefined QP peak does not imply that the system has reached the Fermi liquid regime. As Fig. 9 shows, the chemical potential in this intermediate-temperature metal can be quite far from the Fermi energy, despite a signature of well-distinguishable resilient QPs.

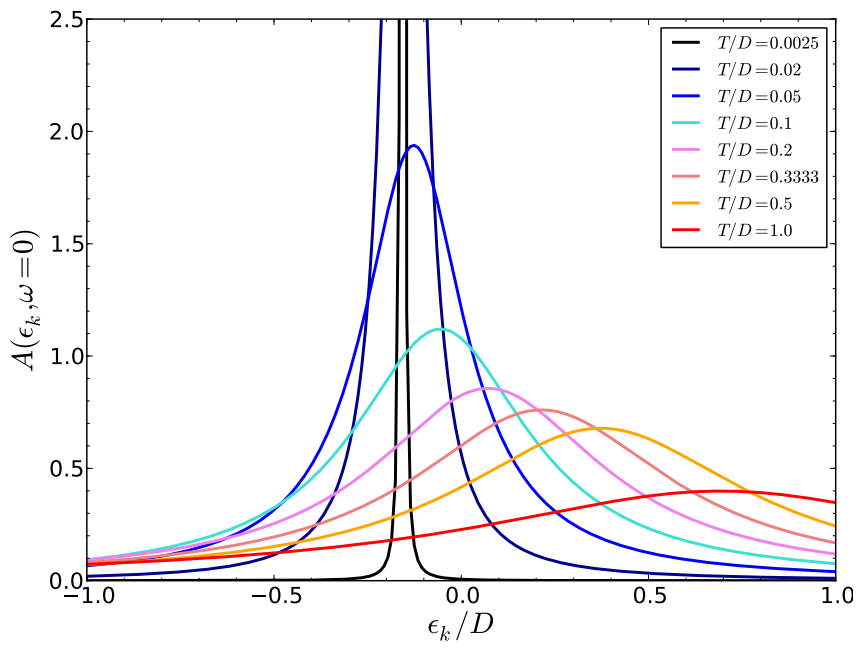

FIG. 8. The evolution of momentum-distribution curves at the Fermi level Eq. A.3. 


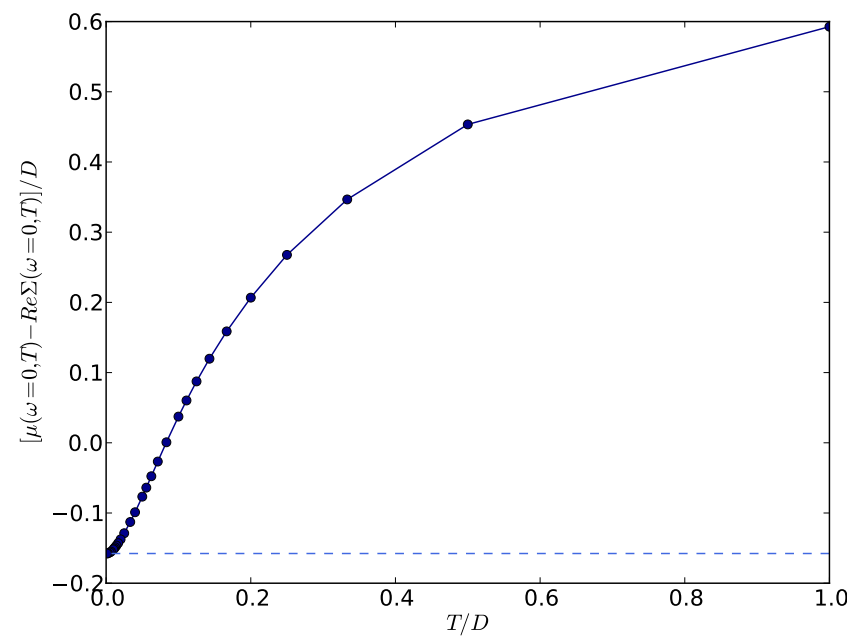

FIG. 9. Temperature dependence of $\mu_{\text {eff }}=\mu(T)-\operatorname{Re} \Sigma(\omega=$ $0, T)$. The noninteracting chemical potential $\mu_{0}$ is shown by a dashed line.

Thermopower at high temperatures and comparison to approximate formulas

In Fig. 10 we show the Seebeck coefficient over a larger temperature window. The Seebeck coefficient calculated using the Kubo formula is plotted with a thick line and compared to various estimates. The Kelvin formula $\partial \mu / \partial T$ (using $\mu$ calculated within DMFT) overestimates the magnitude of the thermopower in the low- $T$ regime but is a good approximation of the exact result above $T_{*} \approx 0.08 D$. For comparison, we also plot the corresponding atomic estimate using the Kelvin formula, but using the $\mu$ obtained in the atomic limit. We note that the two expressions essentially match above $T / D=1$. Note that the Kubo result starts to deviate significantly from the atomic estimate only when entering the resilient QP regime.

Finally, we plot Heikes estimates. The results obtained from the DMFT chemical potential using NRG and continuous-time interaction expansion Monte Carlo (CTINT) are plotted (full green line and symbols). Heikes formula is found to approximate the thermopower worse than the Kelvin formula. For comparison, also the atomic Heikes estimates (thick dashed) as well as the asymptotic $U \rightarrow 0$ and $U \rightarrow \infty$ Heikes values (horizontal lines) are shown. 


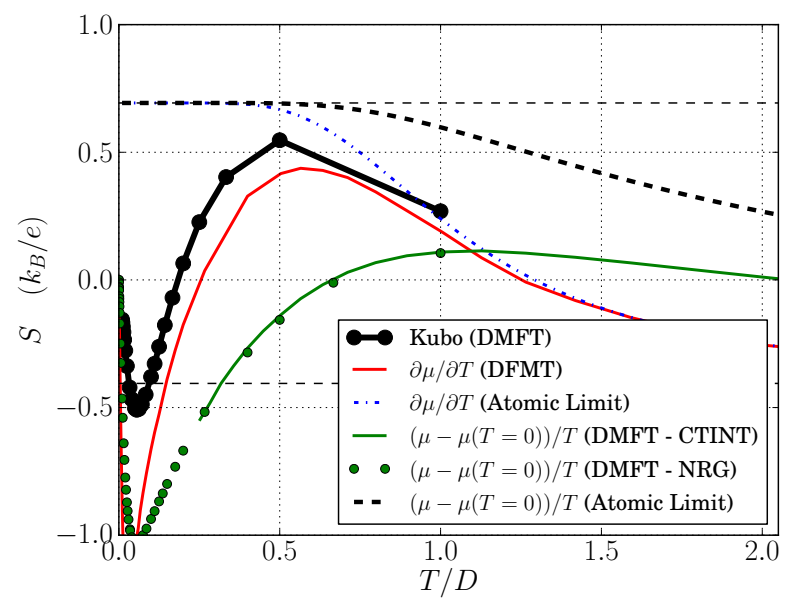

FIG. 10. Seebeck coefficient calculated using the exact Kubo formula (thick black line) compared to the approximate Kelvin formula (red line) and Heikes formula (green line and green symbols). The atomic Kelvin estimate (dotted) and Heikes estimate (thick dashed) interpolate between the asymptotic $U \rightarrow \infty$ and $U \rightarrow 0$ Heikes values. 